\title{
Two-Dimensional Sideband Raman Cooling and Zeeman State Preparation in an Optical Lattice *
}

\author{
A.V. Taichenachev, A.M. Tumaikin, and V.I. Yudin \\ Novosibirsk State University, Pirogova 2, Novosibirsk 630090, Russia \\ L. Hollberg \\ Time and Frequency Division, National Institute of Standards and Technology, \\ 325 Broadway, MS 847-10, Boulder, CO 80303
}

(September 4, 2018)

\begin{abstract}
A method of sideband Raman cooling to the vibrational ground state of the $m=0$ Zeeman sublevel in a far-detuned two-dimensional optical lattice is proposed. In our scheme, the Raman coupling between vibrational manifolds of the adjacent Zeeman sublevels is shifted to the red sideband due to the ac Stark effect induced by a weak pump field. Thus, cooling and optical pumping to $m=0$ is achieved by purely optical means with coplanar cw laser beams. The optical lattice and cooling parameters are estimated in the framework of simple theoretical models. An application of the transverse sideband cooling method to frequency standards is discussed. Coherent population trapping for the sideband Raman transitions between the degenerate vibrational levels is predicted.
\end{abstract}

PACS: $32.80 . \mathrm{Pj}, 42.50 . \mathrm{Vk}$

\section{INTRODUCTION}

Laser-cooled atoms play a critical role in modern frequency standards such as atomic fountains [1]. As is well-known, Sisyphus-type cooling in optical molasses with polarization gradients results in atoms with temperatures corresponding to tens of the single-photon recoil energies $\varepsilon_{r}=(\hbar k)^{2} / 2 M$ (for example, $T \sim 30 \varepsilon_{r} / k_{B} \sim 3 \mu K$ in the case of $C s$ [2]). Even lower temperatures can be achieved by velocity-selective methods [3 5]. These methods, however, require more complicated technical implementations [- $[$.

Recently, Poul Jessen and co-workers [6] demonstrated an elegant and efficient method of cooling atoms to the vibrational ground state of a far-off-resonance two-dimensional optical lattice. Their method is a variant of Raman sideband cooling [7] based on transitions between the vibrational manifolds of adjacent Zeeman substates. A static magnetic field is used to tune the Zeeman levels so that Raman resonance occurs on the red sideband and results in cooling. Two circularly polarized fields are then used to recycle the atoms for repetitive Raman cooling. The cooling operates in the Lamb-Dicke regime with cw laser beams and does not require phase-locked lasers; a transverse temperature of about $950 \mathrm{nK}$ was achieved.

Stimulated by the concepts and results from Jessen [6], we propose a new variant of transverse sideband cooling. The basic difference from the method of Ref. [6] is that a linearly polarized pumping field, detuned from resonance, now plays a two-fold role. It provides both optical pumping back to the $m=0$ magnetic sublevel, and it causes a uniform ac Stark shift that replaces the external magnetic field-induced Zeeman shift that was used in Ref. [6]. The main improvement consists in the flexibility of optical methods of the atomic state control. For example, in our scheme, changing the polarization from linear to circular and the direction of the pumping beam, we can accumulate all atoms in the outermost Zeeman substate $m=F$.

One promising application of this technique is for two-dimensional cooling of atoms in an atomic fountain, or for a space-based atomic clock. More precisely, the method can be used to cool an atomic sample down to sub- $\mu K$ temperatures in the transverse directions before launching the cold atoms into the Ramsey interaction region. For the long Ramsey periods that could be used in an atomic clock in space, reducing the transverse spread would increase substantially the number of atoms in the detection region. In this context, Jessen's original scheme has some disadvantages. Namely, atoms are accumulated in the stretched $m=F$, substate of the $F=4$ ground-state hyperfine level of $C s$, a perturbing magnetic field is used, and that system also requires that the pumping and repumping beams propagating orthogonal to the cooling plane (i.e. down to axis of the clock), which would perturb atoms drifting through the Ramsey region. Newly proposed atomic-fountain clocks on Earth and clocks proposed for space

${ }^{*}$ Contribution of NIST, not subject to copyright 
will use multiple balls of atoms in the Ramsey region, so there would be atoms in the Ramsey cavity undergoing state interrogation while other atoms are state prepared and detected. In this case the out-of-plane light beams and magnetic field would produce unacceptable frequency shifts. Some of these problems are of a technical character and could be solved by different methods. For instance, atoms might be transfered from $|F=4, m=4\rangle$ to $|F=4, m=0\rangle$ without additional heating by the adiabatic passage technique [8], and the Ramsey region might be adequately isolated from the magnetic field. However, the problem of the light beams down the clock axis is more fundamental problem. Our scheme avoids these problems without additional technical complications, while maintaining most of the attractive features. In particular, in the present version, only cw lasers lying in the cooling plane are used, there is no magnetic field, and atoms are prepared in the $m=0$ substate. Since the linear polarization of the pumping field coincides (must coincide) with the orientation of the clock's magnetic field (C-field) in the Ramsey region, switching between the Stark shifted and Zeeman shifted substates will not produce any mixing.

In the following section we discuss the proposed lattice and cooling parameters in the framework of a simple theoretical model. The optimal magnitudes of the Raman transition amplitude, the pumping field intensity, and the detuning are found. These analytical results are then confirmed by numerical calculations for a more realistic model of the $F \rightarrow F^{\prime}=F$ transition. In addition, we find that coherence between degenerate (or nearly degenerate) lower vibrational levels can lead, under certain conditions, to significant changes in the cooling efficiency and cooling time.

The proposed cooling method may also be useful for atom optics by providing a high-brightness well-collimated source of atoms, or for general purposes of quantum-state control in a non-dissipative optical lattice.

\section{OPTICAL LATTICE}

The field configuration used for the optical lattice consists of three linearly polarized beams having equal amplitudes and propagating in the $x y$-plane with angles of $2 \pi / 3$ between them (Fig. 1). The polarization vectors of these beams are tilted through a small angle $\phi$ with respect to the $z$-axis. The resulting field can be written as

$$
\begin{aligned}
\mathbf{E}(\mathbf{r}, t) & =E_{0} \mathcal{E}(\mathbf{r}) \exp \left(-i \omega_{L} t\right)+\text { c.c } \\
\mathcal{E}(\mathbf{r}) & =\mathbf{e}_{z} \sum_{i=1}^{3} \exp \left(i \mathbf{k}_{i} \mathbf{r}\right)+\tan (\phi) \sum_{i=1}^{3} \mathbf{e}_{i} \exp \left(i \mathbf{k}_{i} \mathbf{r}\right),
\end{aligned}
$$

where $\mathbf{k}_{i}$ and $\tan (\phi) \mathbf{e}_{i}$ are respectively the wave vectors and the in-plane components of the polarization of the $i$-th beam. All the beams have the same frequency, $\omega_{L}$, far-detuned to the red of the $D_{2}$ resonance line.

As was shown in Ref. [9], if the detuning is much greater than the hyperfine splitting of the excited state, then the optical potential for the ground state takes the form

$$
\widehat{U}_{F}=-\frac{2}{3} u_{s}|\mathcal{E}(\mathbf{r})|^{2}+\frac{i}{3} u_{s} g(F)\left[\mathcal{E}(\mathbf{r})^{*} \times \mathcal{E}(\mathbf{r})\right] \cdot \widehat{\mathbf{F}} .
$$

Here $g(F)=[F(F+1)+J(J+1)-I(I+1)] /[F(F+1)]$ where $F, J$ and $I$ are respectively the total, the electron and the nuclear angular momenta of the ground state, and $\widehat{\mathbf{F}}$ is the angular-momentum operator. The single-beam light shift $u_{s}=-A \mathcal{I} / \Delta$, defined as in Ref. [9], is proportional to the single-beam light intensity $\mathcal{I}$ and inversely proportional to the detuning $\Delta=\omega_{L}-\omega_{F, F_{n}^{\prime}}$. For the $D_{2}$ line of ${ }^{133} \mathrm{Cs}$ the constant $A \approx 1.5 \varepsilon_{r} \mathrm{GHz} /\left(\mathrm{mW} \cdot \mathrm{cm}^{-2}\right)$.

To zeroth order in $\tan (\phi) \ll 1$, the field (11) is linearly polarized along $\mathbf{e}_{z}$ everywhere and vector term in Eq. (2) vanishes, resulting in the isotropic optical potential

$$
\widehat{U}^{(0)}=-\frac{4}{3} u_{s}\left[\frac{3}{2}+\cos (\sqrt{3} k x)+\cos \left(\frac{\sqrt{3} k x-3 k y}{2}\right)+\cos \left(\frac{\sqrt{3} k x+3 k y}{2}\right)\right] .
$$

In other words, contrary to the field configuration of Ref. [6], all the Zeeman sublevels have the same optical shift. For red detunings $\Delta<0$, the minima of the potential (3) form a lattice consisting of equilateral triangles with a side $2 \lambda / 3$ (one of them has the coordinates $x=y=0$ ).

In the general case, the atomic motion in a periodic potential leads to a energy band-structure. However, for potentials with a periodicity of the order of the light wavelength $\lambda$ and with the depth much larger than the recoil energy $\varepsilon_{r}\left(6 u_{s}\right.$ in the case under consideration), both the tunneling probability and the width are exponentially small for bands close to a potential minimum. Hence, instead of a lattice with energy bands we can consider vibrational levels arising from independent potential wells. The spectrum of the lower levels can be defined, with good accuracy, from the harmonic expansion in the vicinity of the well's bottom:

$$
\widehat{U}^{(0)} \approx u_{s}\left[-6+3 k^{2}\left(X^{2}+Y^{2}\right)\right],
$$


where $X$ and $Y$ are the displacements from the minimum. This expansion corresponds to a 2D isotropic harmonic oscillator with the frequency $\hbar \omega_{v}=\sqrt{12 u_{s} \varepsilon_{r}}$. Due to the isotropy, the $n$-th energy level is $n+1$ times degenerate. If the energy separation between adjacent vibrational levels is much greater than the recoil energy, the characteristic size of lower vibrational states is $l=\sqrt{\hbar / M \omega_{v}} \ll \lambda$. In this case we have strong localization, and the Lamb-Dicke regime holds.

\section{SIDEBAND RAMAN COOLING}

Raman transitions between vibrational levels of adjacent magnetic substates are induced by the small in-plane component of the field (11). To first order in $\tan (\phi)$, the vector part of Eqn. (3) gives the correction

$$
\widehat{U}^{(1)}=\frac{1}{3} u_{s} g(F) \tan (\phi) \mathbf{M}(\mathbf{r}) \cdot \widehat{\mathbf{F}},
$$

where $\mathbf{M}$ has the components $M_{x}=2 \sqrt{3}[\cos (3 k y / 2) \sin (\sqrt{3} k x / 2)+\sin (\sqrt{3} k x)]$ and $M_{y}=6 \sin (3 k y / 2) \cos (\sqrt{3} k x / 2)$. Since this term conserves the symmetry of the main potential (3), each well in the lattice obeys the same conditions for the Raman transitions. For the lower vibrational levels we use a first-order approximation with respect to the displacements $X, Y$ from the minimum

$$
\begin{aligned}
\widehat{U}^{(1)} & \approx 3 u_{s} g(F) \tan (\phi) k\left(X \widehat{F}_{x}+Y \widehat{F}_{y}\right) \\
& =\frac{3}{2} u_{s} g(F) \tan (\phi) k\left((X-i Y) \widehat{F}_{+}+(X+i Y) \widehat{F}_{-}\right),
\end{aligned}
$$

where $\widehat{F}_{ \pm}$are the standard raising and lowering angular momentum operators. The operator $\widehat{U}^{(1)}$ has off-diagonal elements both for the vibrational and for the magnetic quantum numbers, inducing transitions with the selection rules $\Delta n= \pm 1$ and $\Delta m= \pm 1$ (for a quantization axis along $\mathbf{e}_{z}$ ). In order of magnitude, the Raman transition rate between the lower vibrational levels is $U_{R}=u_{s} \tan (\phi) k l$. As was shown in Refs. [9, 6], sideband cooling and coherent quantum-state control require this rate to be much greater than the spontaneous scattering rate of lattice photons $\gamma_{s}=6 \Gamma u_{s} / \Delta$, where $\Gamma$ is the natural width. In our lattice $U_{R} / \gamma_{s} \approx 0.2 \tan (\phi) \Delta / \Gamma\left(\varepsilon_{r} / u_{s}\right)^{1 / 4}$.

Two other important requirements for efficient Raman sideband cooling are a spatially independent energy shift of the magnetic sublevels and optical pumping. To achieve these, we propose to use another optical field, known as the pump beam, linearly polarized along the $z$-axis, propagating in the cooling plane, and detuned by several $\Gamma$ to the blue of the $F \rightarrow F^{\prime \prime}=F$ transition of the $D_{1}$ line [10] (Fig. 1). In this case the $m=0$ sublevel is dark and unshifted, while the others undergo the light shifts

$$
\delta_{m}=m^{2} \frac{\Delta_{p} \Omega_{p}^{2}}{\Gamma^{2} / 4+\Delta_{p}^{2}}
$$

where $\Omega_{p}$ is the Rabi coupling for the $|F, m= \pm 1\rangle \rightarrow\left|F^{\prime \prime}=F, m^{\prime \prime}= \pm 1\right\rangle$ transitions and $\Delta_{p}$ is the detuning of the pump field. With a proper choice of $\Omega_{p}$ and $\Delta_{p}$, the states $|m=0, n+1\rangle$ and $|m= \pm 1, n\rangle$ will have the same energy, which leads to efficient transition between them due to the Raman coupling. The cooling picture is completed by the optical pumping, which provides the relaxation from $|m= \pm 1, n\rangle$ to $|m=0, n\rangle$ (see Fig. 2.a). The vibrational quantum number $n$ is conserved in this process due to the fact that atoms are in the Lamb-Dicke regime. It is important to note that, contrary to Ref. [6], in our case, several levels are cooled simultaneously due to the isotropy of the potential $\widehat{U}^{(0)}$ (3). If $\omega_{v} \gg U_{R}$ the state $|m=0, n=0\rangle$ is approximately dark and the majority of the atoms are eventually pumped into this target state. Thus, the described cooling method can be viewed as a version of dark-state cooling. Aspects of this cooling scheme that distinguish it from that of Ref. [6] are: the cooling will be more efficient because more levels involved, the final pumping is to dark state $m=0$, and only laser fields in a plane perpendicular to the quantization axis are used.

To insure that there are no additional constraints and to estimate the cooling parameters, we consider a simple theoretical model based on the double $\Lambda$-system (see Fig. 2.b). This simplified case allows an analytical treatment of the problem. We find the steady-state solution to the generalized optical Bloch equations involving the light-induced and spontaneous transitions and the Raman coupling. We are primarily interested in the limits

$$
\Delta_{p} \gg \Gamma ; \omega_{v} \gg U_{r}
$$

because in this case the light-shift exceeds the field broadening and we can shift the states $|2\rangle$ and $|5\rangle$ into degeneracy with negligible perturbation of state $|1\rangle$. Under these conditions, the solution gives the following results: (i) the 
population of the target state $|1\rangle$ is maximal at exact resonance $\Omega_{p}^{2} / \Delta_{p}=\omega_{v}$ (see Fig. 3.a), (ii) on resonance, the total population of the states coupled with light is small, and equal to $\left(U_{R} / \omega_{v}\right)^{2}$, the probability for off-resonance Raman transitions $|1\rangle \rightarrow|6\rangle$ multiplied by a factor 4 , and (iii) the population of state $|2\rangle$ contains two terms: $\pi_{2}=1 / 2\left(U_{R} / \omega_{v}\right)^{2}+1 / 16\left(\gamma_{p} / \omega_{v}\right)^{2}$. The second term is determined by the ratio of the width imposed by light, $\gamma_{p}=\Gamma \Omega_{p}^{2} / \Delta_{p}^{2}$, to the vibrational frequency $\omega_{v}$. As a result, the target state population is close to unity:

$$
\pi_{1} \approx 1-a\left(U_{R} / \omega_{v}\right)^{2}-b\left(\Gamma / \Delta_{p}\right)^{2} .
$$

The coefficients are $a=3 / 2$ and $b=1 / 16$ in the case of the double- $\Lambda$ system model.

We now turn to an estimate of the cooling dynamics. Instead of looking for a temporal solution of the Bloch equations $(d / d t) \rho=\widehat{\mathcal{L}} \rho$ for atomic density matrix $\rho$ and $\widehat{\mathcal{L}}$ the corresponding Liouvillian superoperator, we find the statistically averaged transition time $\tau=\int_{0}^{\infty}(\rho(t)-\rho(\infty)) d t$ [11]. This matrix obeys the equations $\widehat{\mathcal{L}} \tau=\rho(\infty)-\rho(0)$, where $\rho(\infty)$ is the steady-state solution and $\rho(0)$ is the initial distribution (we set $\pi_{1}=\pi_{2}=\pi_{5}=\pi_{6}=1 / 4$ and the other elements equal to zero at $t=0$ ). The cooling rate can be associated with the inverse transition time for the $|1\rangle$ state $\gamma_{c o o l}=\tau_{1}^{-1}$. As a function of the optical frequency shift, the cooling rate is a Lorentzian curve with a width $\sim \sqrt{1 / 4 \gamma_{p}^{2}+7 U_{R}^{2}}$ (see Fig. 3.a). Exactly on resonance, and in the limits (6) $\Delta_{p}=\Omega_{p}^{2} / \omega_{v}$, and $\gamma_{\text {cool }}$ takes the form

$$
\gamma_{\text {cool }}=\alpha \frac{\gamma_{p} U_{R}^{2}}{\gamma_{p}^{2}+\beta U_{R}^{2}} .
$$

Calculations within the framework of the double $\Lambda$-system give $\alpha=8$ and $\beta=28$. This dependence of the cooling rate on $\gamma_{p}$ and $U_{R}$ can be understood qualitatively if we consider the cooling as optical pumping into the dark state. Obviously, because other parameters do not appear in the conditions (6), the cooling rate is determined entirely by the optical pumping rate $\gamma_{p}$ and the Raman transitions rate $U_{R}$. If $U_{R} \gg \gamma_{p}$, an atom passes from $|2\rangle$ to $|5\rangle$ very quickly, and the cooling rate is proportional to the rate of the slower process of repumping from $|5\rangle$ to $|1\rangle$. In the inverse limit $U_{R} \ll \gamma_{p}$, the slowest process is the transition $|2\rangle \rightarrow|5\rangle$. The corresponding rate, however, is not equal to $U_{R}$, but is suppressed by the factor $U_{R} / \gamma_{p}$. That can be explained as the inhibition of quantum transitions due to continuous measurements on the final state $|5\rangle$ (quantum Zeno effect [12). The cooling rate $\gamma_{\text {cool }}$ as a function of $\Omega_{p}$ (on resonance) is shown in Fig.3.b; $\gamma_{\text {cool }}$ achieves a maximum $\gamma_{\text {cool }}^{\max }=U_{R} \alpha /(2 \sqrt{\beta})$ at the optimal Rabi coupling $\Omega_{p}^{o p t}=(\beta)^{-1 / 4} \omega_{v} \sqrt{\Gamma / U_{R}}$.

The above described laws for the target-state population and for the cooling dynamics are confirmed by numerical calculations for a more realistic model of the $F \rightarrow F^{\prime}=F$ cycling transition with a limited number of vibrational levels of the 2D oscillator taken into account. More precisely, we consider the systems of equations for the steadystate density matrix $\rho(\infty)$ and for the transition time matrix $\tau$ taking into account for all possible coherences and populations. Including all terms gives a large system of algebraic equations, which can be solved for a fixed set of parameters by numerical methods. We use the LU decomposition method. The numerical results are fitted by the formulae (7, 8 ) very well. The fitting coefficients $a, b, \alpha$ and $\beta$ depend on the angular momentum $F$ and on the initial distribution among the vibrational levels. The results, corresponding to the three lowest vibrational levels (with initially equal populations), are presented in Table 1.

In principle, two factors limit the number of vibrational levels which participate efficiently in the cooling: both the anharmonicity and the violation of the Lamb-Dicke regime become appreciable for higher vibrational levels. The second factor is the more stringent limitation and gives $n^{*} \approx 0.1 \hbar \omega_{v} / \varepsilon_{r}$ as an estimate for the maximal vibrational number.

It should be noted that a stray magnetic field can limit the cooling efficiency by breaking the level degeneracy. To be negligible, the Zeeman shift due to any stray magnetic field should be less than the Raman transition rate $U_{R}$, or the width imposed by the pumping field $\gamma_{p}$, which are of the same order of magnitude for optimal cooling.

To provide the cyclic interaction of the atoms with the pump field, repumping from the other hyperfine level is necessary. For this we propose using another light beam tuned in resonance with a $F \rightarrow F^{\prime}=F+1$ transition of the $D_{2}$ line. This beam is linearly polarized along $e_{z}$ and runs in the $x y$-plane. For example, if the pumping field operates on the $F=4 \rightarrow F^{\prime \prime}=4$ of the $D_{1}$ line of $C s$, the repumping field is applied to the $F=3 \rightarrow F^{\prime}=4$ transition of the $D_{2}$ line. To minimize effects of optical pumping on the other hyperfine level, the intensity of the repumping field should be chosen close to the saturation intensity. It is noteworthy that in our lattice the potentials for both hyperfine levels have the same spatial dependence, and consequently the requirement on the repump intensity is not as stringent as in Ref. [6].

For purposes of illustration we can give numerical estimations for ${ }^{133} \mathrm{Cs}\left(\Gamma \approx 2 \pi 5 \mathrm{MHz}\right.$ and $\left.\varepsilon_{r} / \hbar \approx 2 \pi 2 k H z\right)$. If we take the lattice beams detuning, $\Delta=-2 \pi 10 \mathrm{GHz}$ (from the $F=4 \rightarrow F^{\prime}=5$ transition of the $D_{2}$ line) and intensity $\mathcal{I}=500 \mathrm{~mW} / \mathrm{cm}^{2}$, then the single-beam Stark shift $u_{s} \approx 75 \varepsilon_{r} \approx 2 \pi \hbar 150 \mathrm{kHz}$. The lattice has the 
depth $6 u_{s}=450 \varepsilon_{r} \approx 2 \pi \hbar 900 \mathrm{kHz}$, and thus supports approximately 15 bound levels with the energy separation $\hbar \omega_{v}=30 \varepsilon_{r} \approx 2 \pi \hbar 60 k H z$. With the tilt angle $\tan (\phi) \approx 0.1$, the Raman transition rate is $U_{R} \approx 0.1 \hbar \omega_{v}$, providing the figure of merit $U_{R} / \gamma_{s} \approx 12 \gg 1$. The stray magnetic field must be controlled to the level of a few $m G$ or less. In the case of $C s$ the pumping field should be applied to the $F=4 \rightarrow F^{\prime \prime}=4$ transition of the $D_{1}$ line. The repumping field should be tuned to resonance on the $F=3 \rightarrow F^{\prime}=4$ transition of the $D_{2}$ line, and have an intensity $\sim 10 \mathrm{~mW} / \mathrm{cm}^{2}$ in order to saturate the transitions from all Zeeman sublevels. The optimal pumping field detuning $\Delta_{p} \approx 0.2 \Gamma \omega_{v} / U_{R} \approx 2 \Gamma$ and intensity $\mathcal{I}_{p} \approx 8 \mathrm{~mW} / \mathrm{cm}^{2}$ give the cooling rate $\gamma_{\text {cool }} \approx 0.4 U_{R} \approx 2 \pi 2.2 \mathrm{kHz}$, and time of $\tau \approx \gamma_{\text {cool }}^{-1} \approx 10^{-4} s$. For these conditions our analysis predict approximately $95 \%$ of the population will accumulate in the target state $|F=4, m=0, n=0\rangle$ with lower levels having vibrational numbers up to $n^{*} \approx 0.1 \hbar \omega_{v} / \varepsilon_{r} \approx 3$.

\section{COHERENCE BETWEEN VIBRATIONAL LEVELS}

In the case of a symmetric field configuration for 2D and 3D lattices a degeneracy of the vibrational energy level structure occurs. For a 2D lattice (for example the field configuration of Ref. [6] and our configuration) in the harmonic approximation, the $n$-th vibrational level contains $n+1$ sublevels $\left\{\left|m, n_{x}+n_{y}=n\right\rangle\right\}$. We find that the coherence induced between the degenerate or near-degenerate vibrational levels can play an important role, and significantly change the efficiency of the resonance Raman coupling. For instance, consider a field configuration that differs from the scheme presented in Fig. 1.a by the direction and polarization of the pumping beam. Let this circularly polarized beam propagate along the $z$-axis as is shown in Fig. 4.a. In this case atoms are optically pumped to the outermost Zeeman substate $|m=F\rangle$ (see in Fig. 4.b). If we consider two degenerate vibrational levels, for example $\left|m=F, n_{x}=1, n_{y}=0\right\rangle$ and $\left|m=F, n_{x}=0, n_{y}=1\right\rangle$ coupled by Raman transitions with the unique state $\left|m=F-1, n_{x}=n_{y}=0\right\rangle$ (Fig. 5.a), we find that there exists a superposition of the states of the degenrate vibrational level

$$
\left|\Psi_{N C}\right\rangle=\left|m=F, n_{x}=0, n_{y}=1\right\rangle+i\left|m=F, n_{x}=1, n_{y}=0\right\rangle
$$

that is not coupled with $\left|m=F-1, n_{x}=n_{y}=0\right\rangle$ by the operator $\widehat{U}^{(1)}$ (5): $\widehat{U}^{(1)}\left|\Psi_{N C}\right\rangle=0$. Hence, part of the population will be trapped in this superposition state, in an analogy with well-known coherent population trapping in the $\Lambda$-scheme [13]. The result of such trapping is that the cooling efficiency is reduced, i.e. the part of atoms accumulated in the target state $\left|m=F, n_{x}=n_{y}=0\right\rangle$ will be significantly less than unity. In the case of coupling between higher levels $\left|m=F, n_{x}+n_{y}=n\right\rangle$ and $\left|m=F-1, n_{x}+n_{y}=n-1\right\rangle$, there always exists a coherent superposition of the sublevels $\left|m=F, n_{x}+n_{y}=n\right\rangle$, for which the operator of the Raman transitions is equal to zero, as it is for the light-induced $\Lambda$-chains [14]. However, for higher vibrational levels the anharmonicity has to be taken into account and the degeneracy is partially broken. If the vibrational energy levels are only nearly degenerate, due to either the anharmonicity or the optical lattice asymmetry, dark superpositions are not completely stable due to small energy splitting and atoms pass eventually from $\left|m=F, n_{x}+n_{y}=n\right\rangle$ to $\left|m=F-1, n_{x}+n_{y}=n-1\right\rangle$. But the corresponding transfer time will be greater than the normal $U_{R}^{-1}$, leading to an increase of cooling time.

In the scheme presented in Fig. 1.a, this unwanted coherence effect is avoided by the simultaneous Raman coupling of the two states $\left|m=0, n_{x}+n_{y}=1\right\rangle$ of the degenerate vibrational level, with the two other states $\left|m= \pm 1, n_{x}=n_{y}=0\right\rangle$ with different amplitudes, as is shown in Fig. 5.b. It can be seen from this figure that the dark superposition for the transition with $\Delta m=-1$ is

$$
(X+i Y) \widehat{F}_{-}\left|\Psi_{N C}^{(-)}\right\rangle=0, \quad\left|\Psi_{N C}^{(-)}\right\rangle=\left|m=0, n_{x}=0, n_{y}=1\right\rangle+i\left|m=0, n_{x}=1, n_{y}=0\right\rangle .
$$

While for the transition with $\Delta m=+1$ the uncoupled state is given by

$$
(X-i Y) \widehat{F}_{+}\left|\Psi_{N C}^{(+)}\right\rangle=0, \quad\left|\Psi_{N C}^{(+)}\right\rangle=\left|m=0, n_{x}=0, n_{y}=1\right\rangle-i\left|m=0, n_{x}=1, n_{y}=0\right\rangle .
$$

The states $\left|\Psi_{N C}^{(-)}\right\rangle$and $\left|\Psi_{N C}^{(+)}\right\rangle$are orthogonal, so no a superposition can be made which nullifies the Raman coupling operator $\widehat{U}^{(1)}(5)$. We also note that the coherence within the vibrational structure might be very useful for other purposes, for instance in quantum state preparation.

\section{CONCLUSION}

Concluding, we have proposed a new scheme for 2D Raman-sideband-cooling to the zero-point energy in a faroff-resonance optical lattice and simultaneously pumping atoms to the $m=0$ dark state. The main distinguishing 
features of our proposal is that the method uses a pumping field to Stark-shift the Raman coupling to the red sideband and thus accumulation cold atoms in the $m=0$ Zeeman sublevel. An elementary theoretical consideration allowed us to calculate the dependence for the cooling efficiency and for the cooling dynamics. Our estimates for a cold $C s$ show that for easily realizable experimental parameters up to $95 \%$ of atoms can be accumulated in the $|F=4, m=0, n=0\rangle$ state in a millisecond time scale. This corresponds to a kinetic temperature on the order of $100 \mathrm{nK}$ after adiabatic release from the lattice [15]. Besides, coherent population trapping between degenerate vibrational levels has been predicted for the sideband Raman transitions.

The authors thank Dr. J. Kitching and Prof. P. Jessen for helpful discussions. This work was supported in part by the Russian Fund for Basic Research (Grant No. 98-02-17794). AVT and VIYu acknowledge the hospitality of NIST, Boulder.

[1] J. J. Bollinger, J. D. Prestage, W. M. Itano, and D. J. Wineland, Phys. Rev. Lett., 54, 1000 (1985); M. A. Kasevich, E. Riis, S. Chu, and R. G. DeVoe, Phys. Rev. Lett., 63, 612 (1989); K. Gibble and S. Chu, Phys. Rev. Lett., 70, 1771 (1993).

[2] C. Salomon, J. Dalibard, W. D. Phillips, A. Clairon, and S. Guellati, Europhys. Lett., 12, 683 (1990).

[3] A. Aspect, E. Arimondo, R. Kaiser, N. Vansteenkiste, and C. Cohen-Tannoudji, Phys. Rev. Lett., 61 (1988) 826.

[4] M. Kasevich and S. Chu, Phys. Rev. Lett.,69, 1741 (1992).

[5] J. Lawall, F. Bardou, B. Saubamea, K. Shimizu, M. Leduc, A. Aspect, and C. Cohen-Tannoudji, Phys. Rev. Lett., 73, 1915 (1994).

[6] S. E. Hamann, D. L. Haycock, G. Klose, P. H. Pax, I. H. Deutsch, and P. S. Jessen, Phys. Rev. Lett., 80, 4149 (1998).

[7] D. J. Heinzen and D. J. Wineland, Phys. Rev. A, 42, 2977 (1990); R. Tä̈eb, R. Dum, J. I. Cirac, P. Marte, and P. Zoller, Phys. Rev. A, 49, 4876 (1994); H. Perrin, A. Kuhn, I. Bouchoule, and C. Salomon, Europhys. Lett., 42, 395 (1998).

[8] P. Pillet, C. Valentine, R.-L. Yuan, and J. Yu, Phys. Rev. A, 48, 845 (1993).

[9] I. H. Deutsch and P. S. Jessen, Phys. Rev. A, 57, 1972 (1997).

[10] We propose to use the $D_{1}$ line in order to avoid any interference with the repumping and lattice beams, which operate on the $D_{2}$ line.

[11] This method is a variant of a statistical consideration of a dynamical system first introduced in L. S. Pontryagin, A. A. Andronov, and A. A. Witt, Zh. Eksp. Teor. Fiz., 3, 165 (1933).

[12] W. M. Itano, D. J. Heinzen, J. J. Bollinger, and D. J. Wineland, Phys. Rev. A, 41, 2295 (1990).

[13] E. Arimondo, in Progress in Optics, edited by E. Wolf (North-Holland, Amsterdam, 1996), V. XXXV, p.259.

[14] V. S. Smirnov, A. M. Tumaikin, and V. I. Yudin, Sov. Phys. JETP, 69, 913, (1989).

[15] A. Kastberg, W. D. Phillips, S. L. Rolston, R. J. C. Spreeuw, and P. Jessen, Phys. Rev. Lett., 74, 1542 (1995).

TABLE I. The fitting parameters for different transitions $F \rightarrow F^{\prime \prime}=F$.

\begin{tabular}{|c|c|c|c|c|}
\hline$F$ & $a$ & $b$ & $\alpha$ & $\beta$ \\
\hline$\overline{\overline{1}}$ & "3.0 & 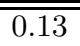 & 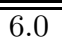 & $\overline{\overline{27}}$ \\
\hline 2 & 2.6 & 0.13 & 4.6 & 27 \\
\hline 3 & 2.5 & 0.13 & 4.1 & 27 \\
\hline 4 & 2.4 & 0.13 & 3.8 & 27 \\
\hline
\end{tabular}



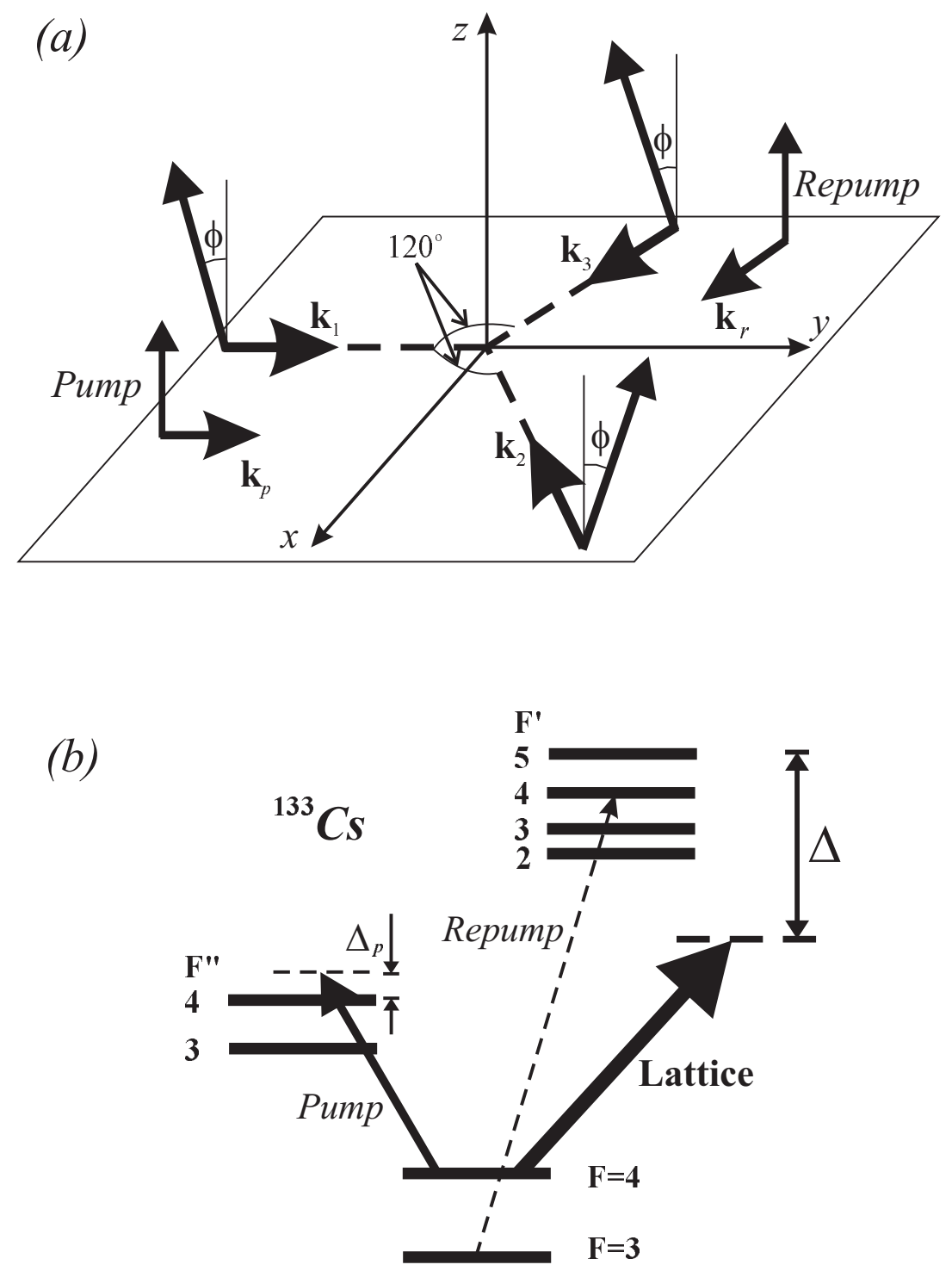

Fig.1

FIG. 1. (a) Field geometry. The basic optical lattice is formed by three coplanar beams that are linearly polarized along the $z$-axis. The small in-plane component of polarizations (the tilt angle is indicated by $\phi$ ) induces the Raman coupling. The pumping and repumping beams also propagate in the $x y$-plane and have $e_{z}$ linear polarization. (b) The field detunings for the case of ${ }^{133} \mathrm{Cs}$ are: the lattice beams are far-detuned to the red side of the $F=4 \rightarrow F_{\max }^{\prime}=5$ transition of the $D_{2}$ line, the pumping field is tuned to the blue side of the $F=4 \rightarrow F^{\prime \prime}=4$ transition of the $D_{1}$ line, and the repumping field is tuned in resonance with the $F=3 \rightarrow F^{\prime}=4$ transition of the $D_{2}$ line. 


\section{(a)}
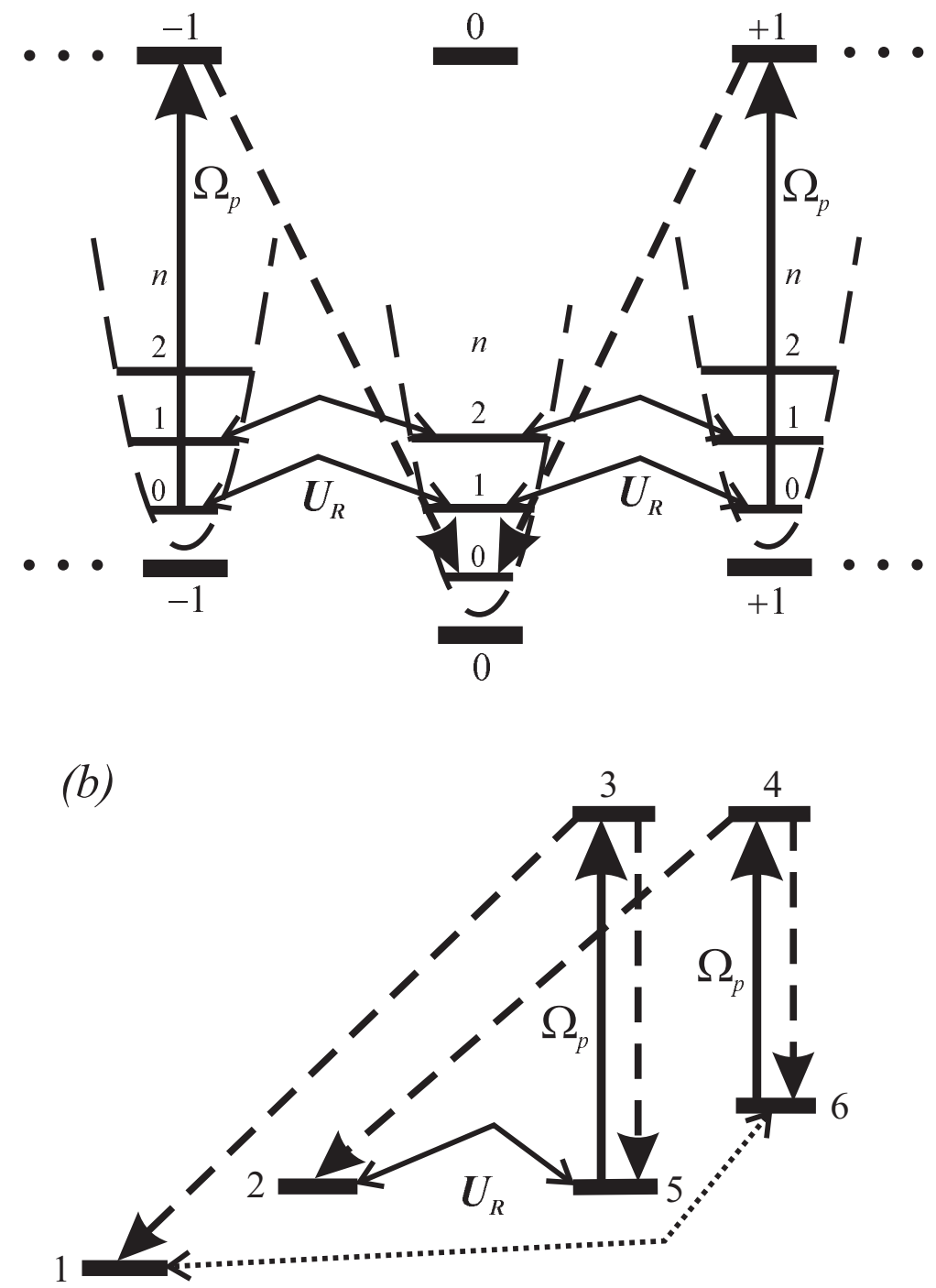

Fig.2

FIG. 2. (a) Scheme of the sideband Raman cooling. Raman transitions are shown by tilted arrows, connecting $|m=0, n\rangle$ to $\left|m= \pm 1, n^{\prime}=n-1\right\rangle$ states. The transitions induced by the pumping field (solid lines) and spontaneous transitions (dashed lines) provide the relaxation back to the $|m=0, n=0\rangle$ state. The Zeeman sublevels are shown shifted by the optial Stark effect of the pump laser field. (b) Simple double- $\Lambda$ system model. The states $|1\rangle,|3\rangle$, and $|5\rangle$ are within the odd $\Lambda$-system and are connected by the radiative transitions, which are shown by solid lines. Similarly, the radiative transitions within the even set $|2\rangle,|4\rangle,|6\rangle$ are shown by dashed lines. Transitions between the $\Lambda$-systems $|2\rangle \leftrightarrow|5\rangle$ and $|1\rangle \leftrightarrow|6\rangle$ are both caused by the Raman coupling. (The dots indicate the off-resonant transitions $|1\rangle \leftrightarrow|6\rangle$ ) 

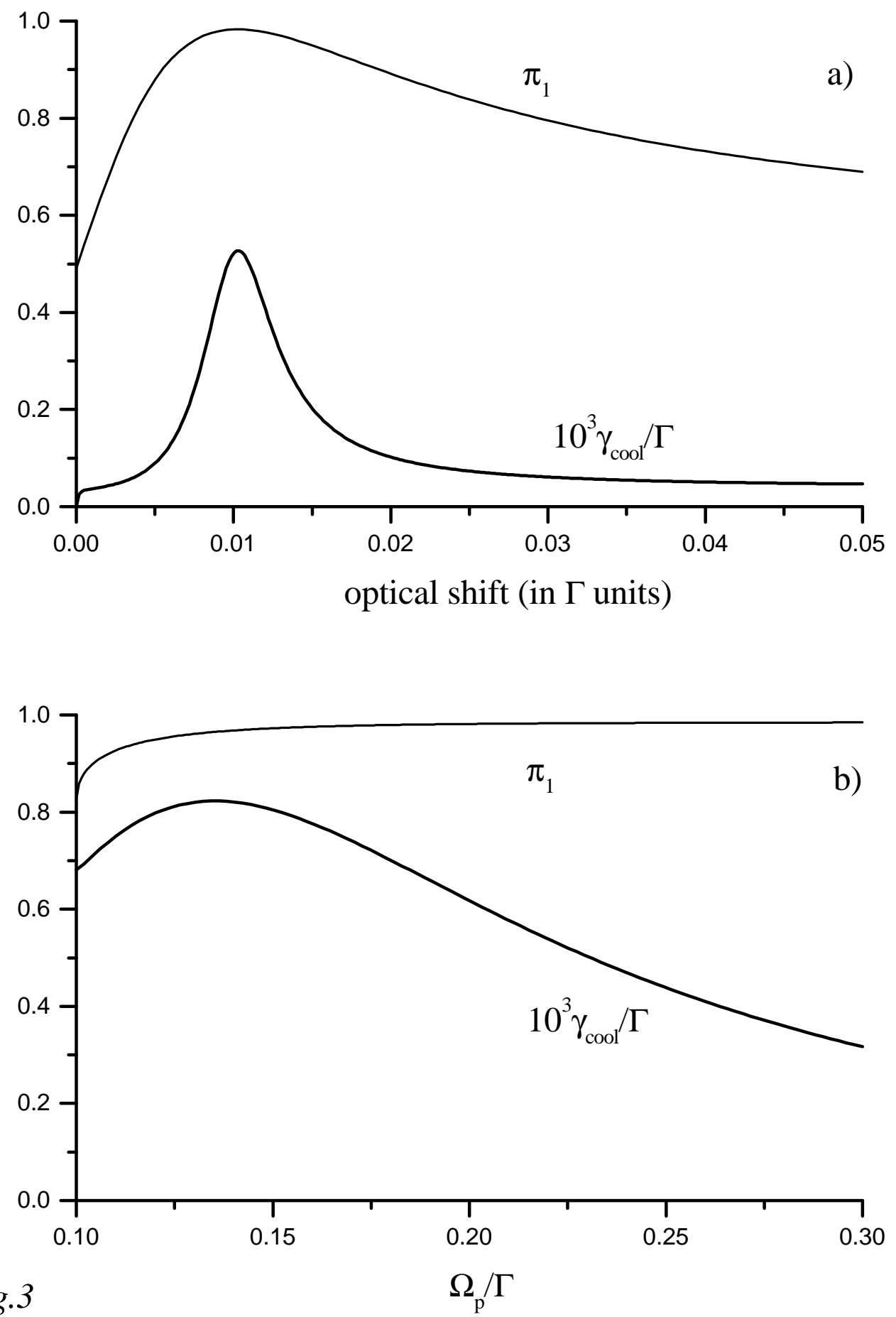

FIG. 3. (a) The target state population $\pi_{1}$ and the cooling rate are plotted versus the optical shift in the case of the double- $\Lambda$ system model for parameters $\Delta_{p}=5 \Gamma, \omega_{v}=0.01 \Gamma$ and $U_{R}=0.001 \Gamma$. (b) The target state population $\pi_{1}$ and the cooling rate are plotted versus the Rabi frequency for exact resonance. The parameters are $\omega_{v}=0.01 \Gamma$ and $U_{R}=0.001 \Gamma$. 


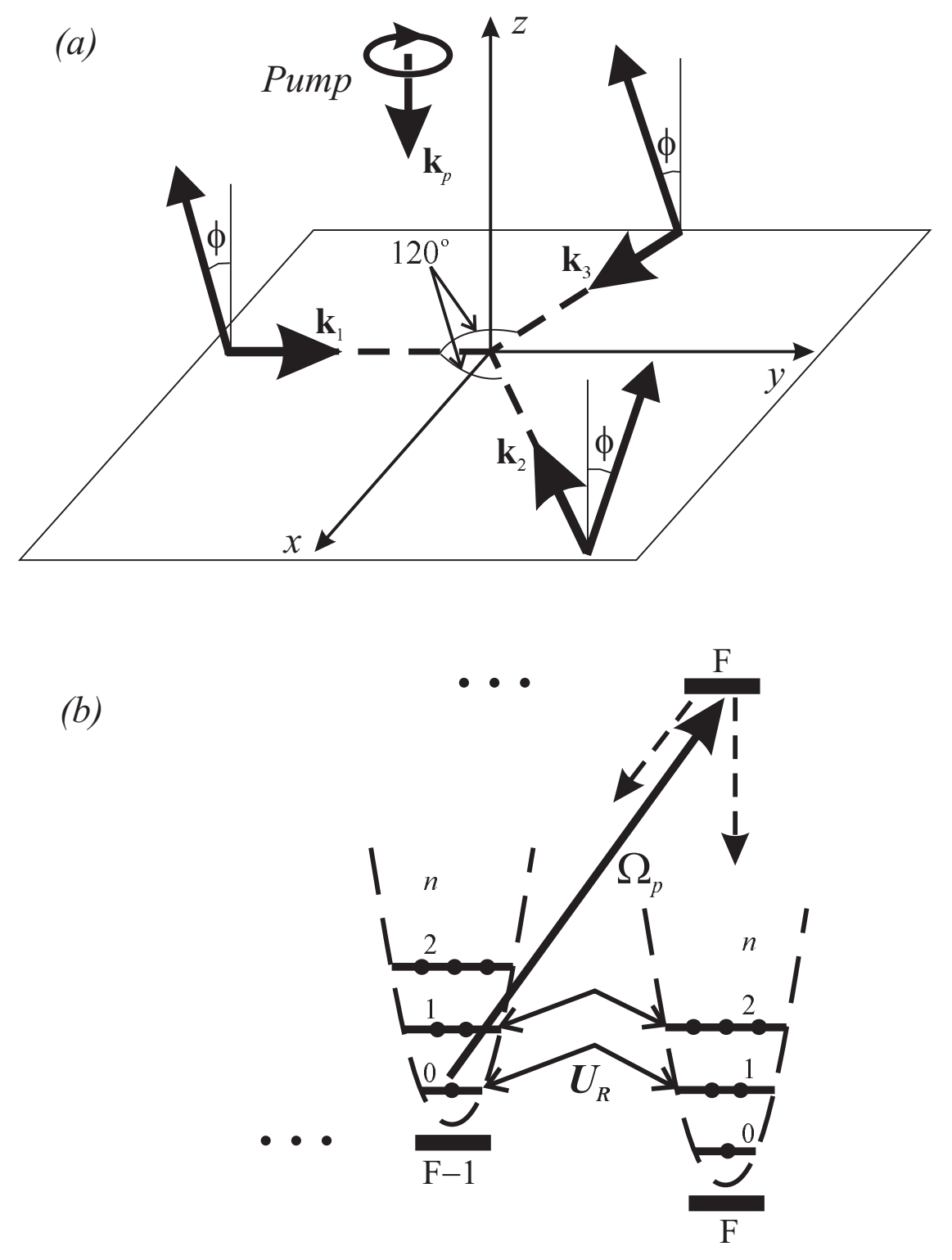

Fig.4

FIG. 4. (a) Field configration for the $m=F$ Zeeman state preparation. It differs from the scheme shown in Fig. 1.a by the polarization and direction of the pumping beam. (b) The light-induced, spontaneous, and Raman transitions between the vibrational levels of Zeeman substates for this case. The small circles on the vibrational levels indicate the degree of degeneracy. 
(a)

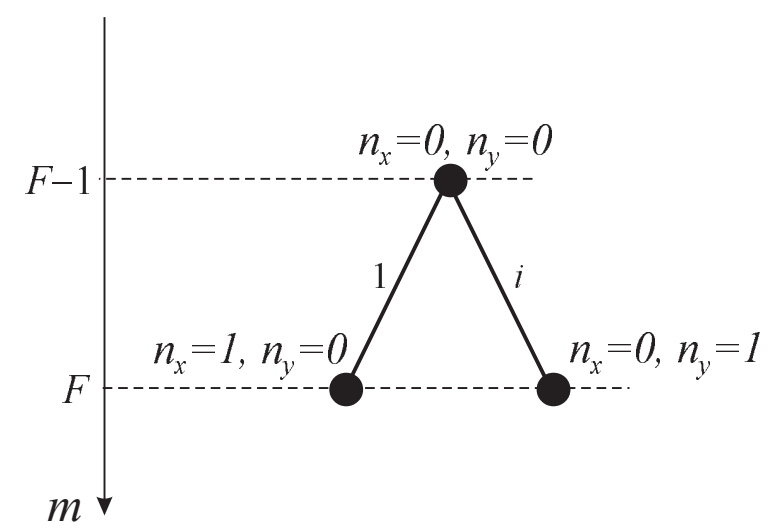

(b)

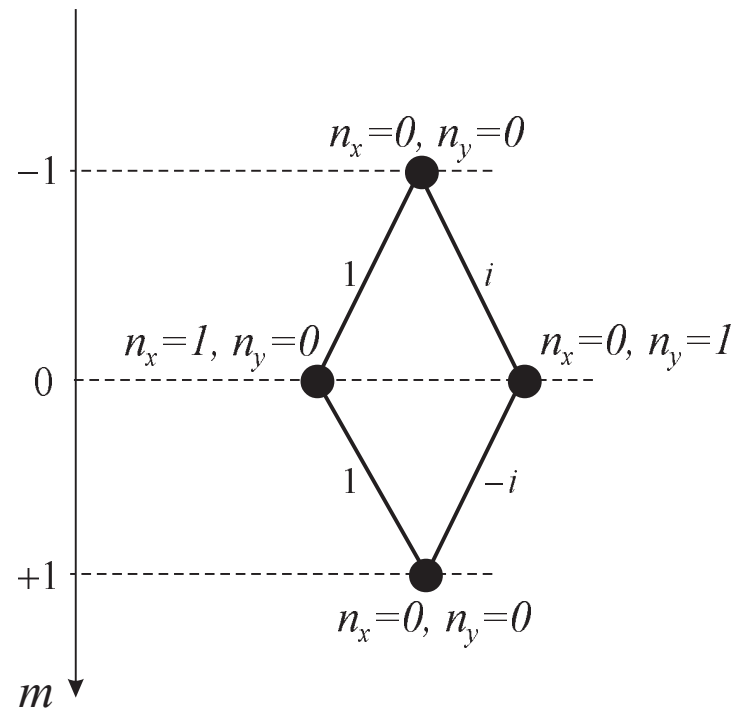

Fig.5

FIG. 5. Scheme of Raman coupling between the lower vibrational levels of adjacent Zeeman substates taking into account the degeneracy (a) in the case of the $m=F$ state preparation, and (b) in the case of the $m=0$ state preparation. The relative amplitudes of the Raman transitions (solid lines) are indicated. 